\title{
STUDIES IN DISSOCIATION
}

\section{Changes in the Auditory Threshold Induced BY 'CRYstal Gazing' 1 \\ BY LEE E. TRAVIS}

The State University of Iorod

\section{INTRODUCTORY}

\section{Statement of the Problem}

Recently there has been awakened a keen interest in the subconscious, abstraction, dissociation and related topics. These terms have often been used in a loose manner for the simple reason that so little is known about the phenomena which they purport to represent. We know very clearly what we mean by dissociation when the spinal cord is severed. Here the higher centers are actually separated from the lower spinal centers and the cortico-spinal neurones can no longer influence the motor responses determined by the impulse from the anterior horn cells. Since the reflexes are exaggerated under such conditions we can assume that the cerebral motor centers prevent the free play of simple reflexes.

The question arises as to whether voluntary abstraction or dissociation is at all related to actual operative dissociation. Can an individual voluntarily remove in any degree the inhibitory effect of cortical control and become a reflex machine? If all individuals cannot make this dissociation, are there some abnormal individuals who can do so? It is believed that these are fair questions to ask and the group of experiments that have been started, the first one of which is here reported, are being undertaken in an attempt to answer them.

This first report is a study of the changes in the auditory threshold for a relatively pure tone while 'crystal gazing.'

1 This paper reports an experimental study carried on in the laboratory of the Psychological Clinic at the State University of Iowa under the direction of Professor John J. B. Morgan, Director of the Clinic. 


\section{Previous Work}

There is very little to be found in the literature regarding the threshold of hearing during states of abstraction and mild dissociation. Dunlap' made some experiments on 'the effect of physical interruptions in subliminal phases,' which, although conducted from an altogether different point of view, hinted at the possibility of the threshold changing under certain conditions. His findings were the results of the summation effect. He used a subliminal sound of constant pitch, and the observers were to respond at the instant of the appearance and disappearance of the sound by predetermined clicks. In his experiments no attempts were made to alter the state of the observer after the normal threshold had been obtained.

Various writers have undoubtedly supposed that changes in the thresholds of the different senses occur under certain conditions and especially in states of hypnotism and somnambulism. Most of the evidence for such suppositions has been gathered from pathological cases, and suggests the need of carefully controlled experiments on normal subjects.

\section{Method and Apparatus}

The individuals who acted as observers for this investigation were either juniors, seniors or graduate students in the State University of Iowa.

The main piece of apparatus used in the investigation was the fourth model of the Iowa Pitch-Range Audiometer, ${ }^{2}$ about which only a few facts will be presented in order to make the experiments clear. The tone generated is comparatively pure and can be varied in pitch from $30 \mathrm{v}$. d. to over 7,000 v. d. A tachometer is so calibrated that the pitch being generated can be read at any instant. The vibration frequency can be changed according to the desire of the experimenter by means of a rheostat which is connected in 308.

${ }^{1}$ Studies from the California Psychological Laboratory, Psychol. Reo., I904, XI.,

2 For a detailed description of this apparatus see paper by C. E. Seashore, Lancet, Oct. 15, 1919. 
series with the motor. This adjustment was not used in this experiment since the pitch was kept constant throughout each experiment. The tone can be produced in the observer's receiver by connecting through a double-pole knife switch.

The resistance and hence the intensity of the tone as heard in the receiver is controlled by a device which consists of a series of twenty-one non-inductive resistances, installed in series with the generator. The first contact represents minimal intensity; according to experimental findings, it is not absolute zero resistance. The second contact has a resistance of $.000025 \mathrm{ohm}$, and the other steps increase throughout the series by increments of $250 \%$, totalling $2,264.3 \mathrm{ohms}$ resistance for the maximal intensity. The loudness of the tone can be varied and designated in terms of these resistances, as each of these contacts becomes a connection with the watch case receiver terminals when the switch is shifted from one to the other.

The remainder of the apparatus consists of a clear crystal for gazing about $10 \mathrm{~cm}$. in diameter, mounted over a 40-watt blue, mazda light bulb; a silent signal key; and a watch case receiver fastened to a head-clamp. The apparatus just described is in the light-proof, sound-proof, and jar-proof room of the psychological laboratory, while the audiometer is in an adjacent room.

The observer was seated comfortably at a table of average writing height with the watch-case receiver held firmly against one ear by the head-clamp. On the table was the silent signal key and the crystal, both of which could be moved to convenient places by the observer. The mercury key was quite sensitive, but still some effort was necessary to make contact. When contact was made, a buzzer sounded at the elbow of the experimenter. Each subject was instructed to try several positions with regard to key and crystal positions in order to find the easiest and most comfortable one.

The threshold of the observer was obtained under conditions identical with those under which the experiment was conducted except that there was no crystal gazing. The subject was to attend to the tones coming in the receiver 
and respond every time he heard one. It might be well to state here that the threshold was ascertained both before and after the experiment.

The threshold having been found, the light was then switched on beneath the crystal into which the observer was asked to gaze. The crystal gave the appearance of the heavens on a clear, star-lit night. Each individual was told to begin the abstraction by recalling some pleasant experience or imagine himself in a wished-for or cherished situation. If these failed to cause him to forget his present surroundings, he was advised to attempt placing himself into a hypnagogic state. Several of the observers did approach this latter condition. When the subject fully understood what was expected of him, he was again left alone, to gaze into the crystal.

After from three to five minutes signals were given to the observer in series of three, the series at least a half minute apart. The intensity of the beginning tones was generally two contacts below the threshold. If the subject responded to tones of this intensity, the loudness was further decreased by one contact at a time until no responses were received. In case no responses were given on two contacts below normal threshold, the intensity was increased until the observer did respond.

The experiments lasted from one half hour to an hour, depending partly upon results obtained and partly upon the time which could be spent by the individuals acting as observers.

In the table each observer is represented by a capital letter. The thresholds, the tones of certain intensities and the responses to tones of certain intensities are represented by figures; each figure indicating the contact, a larger number being a louder tone. Column five is derived from columns three and four. To make this latter fact clear, let us take observer $D$. His normal threshold before and after the experiment was 14. Tones of intensity 8 were responded to accurately but no responses were made to tone of intensity 7 . Hence his threshold during the experiment in a state of 


\section{Experimental Data.}

TABLE

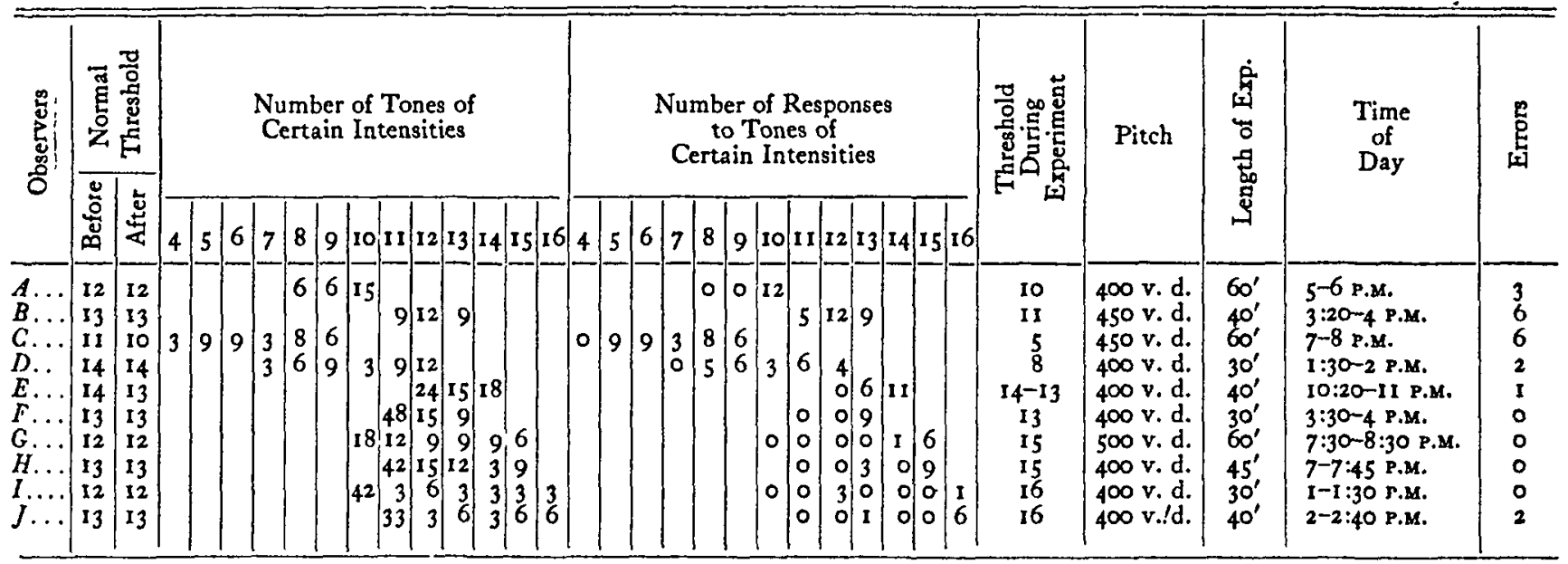

접 
abstraction was 8 . In the cases of observers who required a tone above the normal threshold to provoke a response that tone to which they would react was taken as their threshold during the experiment.

ABOVE

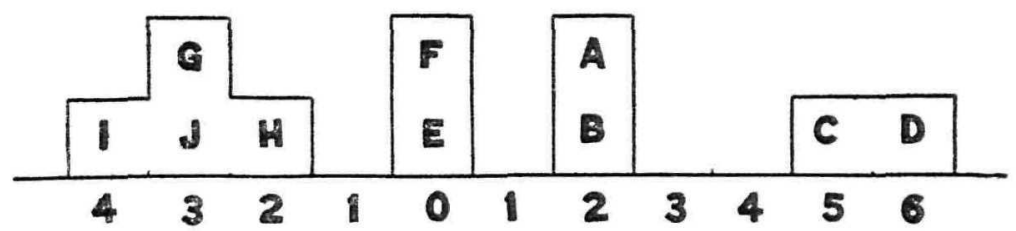
threshold.

Graph I. Distribution of various subjects above and below their normal

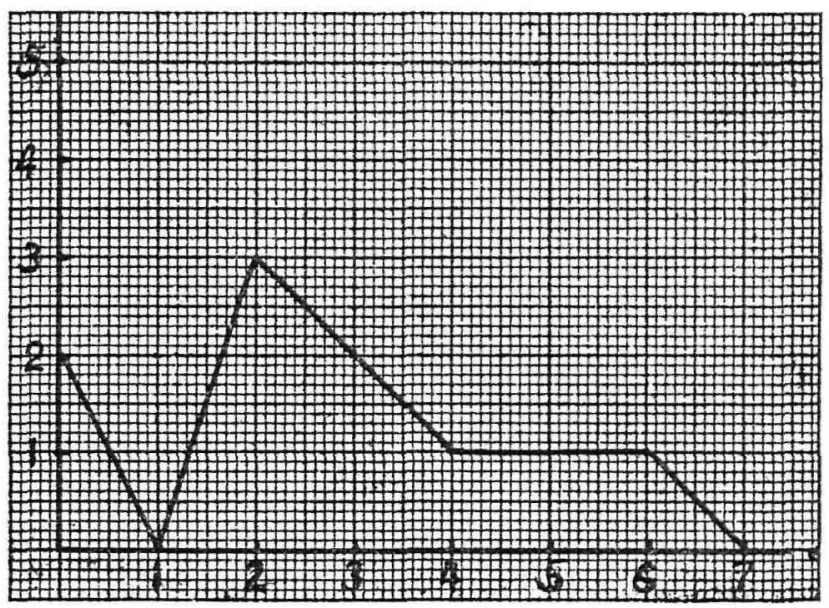

Graph II. Distribution of changes in threshold during abstraction regardless of the direction of the change.

Upon investigation of the table, we find that the normal thresholds of eight out of the ten observers were changed during the experiment. The two whose thresholds were not changed probably were unable to abstract or go into a hypnagogic state. Referring to Graph I., we note that the normal thresholds of four of the observers were lowered, four were raised and two remained unaltered. In other words, four of the observers responded to subliminal stimulation, four re- 
quired a stimulus above the threshold before they would respond and two of the observers reacted to stimuli on the normal threshold. Concerning the two observers whose thresholds remained the same throughout the experiments, nothing can be said except that they undoubtedly did not enter into the state experienced by the others.

One observer's normal threshold was lowered six contacts, each contact being an increase in ohms resistance of 250 per cent. over the previous one. Another's threshold was raised four contacts during the experiment. The other observers fell between these two extremes. Observer $D$ remembered making only two responses when as a matter of fact he made twenty-four. During the experiment he made two false responses.

The case of observer $C$ was slightly different. He did not know whether he responded or not, but knew the tones 'were coming across.' $A$ and $B$ were aroused by the muscular activity involved in pressing the key, when they made responses. A stated that he 'caught himself pushing the key.'

Responding seemed to bring these two observers, $A$ and $B$, back to a normal state and they would not react again to subliminal stimulation until from three to five minutes had elapsed.

All of the observers whose normal thresholds were raised during the experiment stated that when they did hear the tone, it seemed very loud and abrupt. Immediately after a response to a sound which was far above the normal threshold, each of these individuals would respond to tones on the normal threshold and some time had to elapse before he would not react to such stimulation.

\section{INTERPRETATION OF RESULTS}

To give an exact reason why the auditory threshold changes during states of abstraction is not an easy task. The work done in this investigation is too elementary to draw any definite and sharp-cut conclusions. At this time, it will have to suffice merely to give opinions and conjectures. We can only speculate as to why some observers responded 
to subliminal stimulation and others required sounds above the normal threshold to arouse them.

The preliminary practice meager as it was gave the subjects a certain attitude or set. This set carried over into the period of abstraction with four of the subjects so that the responses were made without the awareness of the observer and to stimuli that were below the normal threshold. We cannot be sure whether the difference in response was due to increased acuity of the receiving apparatus or to a facilitation in the nervous conduction brought about by the abstraction. If it were due to increased acuity, it seems that all observers should have reacted alike. If it were due to nervous set, then it is easy to conceive why some acted in one way and some in another. Questioning elicited the confession that those who required a stronger stimulus than their normal threshold tried not to listen for the stimulus; which means that they were set against it and so they required a more intense stimulus to break through this negative set. Those who obeyed the instruction and maintained the set to respond while they abstracted were enabled to react to a stimulus below their normal threshold.

This experiment indicates that the so-called 'automatic' activities that occur in dissociation need not be well practiced habitual responses. The important requirement seems to be that the subject accept the directions of the experimenter. The difference in response may be due to more or less readiness to accept the directions as given, or may be due to the fact that different individuals interpret the same directions differently and so maintain a different set.

\section{Future Research}

The sense of audition presents only one field to study changes in thresholds in states of abstraction. The visual and cutaneous senses are especially susceptible to a study of this sort and should yield as fruitful results as have been obtained in this investigation.

It would be interesting to compare the thresholds of the various senses during 'crystal gazing' with those in a hypnotic 
state. This could be quite easily studied with pathological cases.

Another phase of the problem which would undoubtedly lend itself readily to experimental investigation would be the ascertaining of the reaction time in crystal gazing and compare it with that under ordinary conditions.

After more detailed study has been made, it ought to be possible to standardize a test whereby an individual's ability to voluntarily abstract could be measured. If such a test could be devised, it should prove useful in detecting cases of hysteria.

\section{SUMMARY}

This paper reports an experimental study of the effect of abstraction on the auditory threshold.

I. It was found that the auditory thresholds of eight of the ten observers changed during 'crystal gazing.'

2. The thresholds of four of the individuals were lowered while the thresholds of the other four were raised.

3. The responses made by those observers whose thresholds were lowered were without awareness.

4. When the individuals whose thresholds were raised during the experiment did hear the stimulus, it seemed loud and very abrupt.

5. It has not been sufficiently determined whether the responses to subliminal stimuli were due to increased acuity of the receiving organ or to the neural set involved, although the facts seem to point to the latter interpretation. 